\title{
Trends for prevalence and incidence of resistant hypertension: population based cohort study in the UK 1995-2015
}

\author{
Sarah-Jo Sinnott, ${ }^{1}$ Liam Smeeth, ${ }^{1}$ Elizabeth Williamson, ${ }^{2}$ Ian J Douglas ${ }^{1}$
}

${ }^{1}$ Department of noncommunicable disease

epidemiology, London School

of Hygiene \& Tropical Medicine,

London, WC1E7HT, UK

${ }^{2}$ Department of Medical

Statistics, London School of

Hygiene \& Tropical Medicine,

London, UK

Correspondence to: S-J Sinnott

sarah-jo.sinnott@lshtm.ac.uk

Additional material is published

online only. To view please visit

the journal online.

Cite this as: BMJ 2017;358:j3984 http://dx.doi.org/10.1136/bmj.j3984

Accepted: 17 August 2017

\begin{abstract}
OBJECTIVE

To estimate the incidence and prevalence of resistant hypertension among a UK population treated for hypertension from 1995 to 2015.

DESIGN

Cohort study.

SETTING

Electronic health records from the UK Clinical Practice Research Datalink in primary care.

PARTICIPANTS

1317290 users of antihypertensive drugs with a diagnosis of hypertension.

\section{MAIN OUTCOME MEASURES}

Resistant hypertension was defined as concurrent use of three antihypertensive drugs inclusive of a diuretic, uncontrolled hypertension ( $\geq 140 / 90$ $\mathrm{mm} \mathrm{Hg}$ ), and adherence to the prescribed drug regimen, or concurrent use of four antihypertensive drugs inclusive of a diuretic and adherence to the prescribed drug regimen. To determine incidence, the numerator was new cases of resistant hypertension and the denominator was person years of those with treated hypertension and at risk of developing resistant hypertension. To determine prevalence, the numerator was total number of cases with resistant hypertension and the denominator was those with treated hypertension. Prevalence and incidence were age standardised to the 2015 hypertensive population.
\end{abstract}

\section{WHAT IS ALREADY KNOWN ON THIS TOPIC}

Uncontrolled hypertension is a leading risk factor for morbidity and mortality from cardiovascular disease and stroke

Those with resistant hypertension (uncontrolled hypertension while treated with three antihypertensives inclusive of a diuretic, or those using four antihypertensives) have a higher risk of cardiovascular events than those without resistant hypertension

Current estimates for the epidemiological burden of resistant hypertension are limited by methodological challenges

\section{WHAT THIS STUDY ADDS}

Incidence of resistant hypertension in more than 1.3 million UK primary care patients with hypertension increased from one case per 100 person years in 1996 to a peak of two cases per 100 person years in 2004; thereafter, the incidence decreased to 0.4 cases per 100 person years in 2015

Prevalence increased from $1.75 \%$ in 1995 to a peak of $7.76 \%$ in 2007 then plateaued and declined to $6.46 \%$ in 2015 , reflecting an earlier decline in incidence

Resistant hypertension is common in the UK. Continued effort is needed to reduce the proportion of the population with uncontrolled hypertension given its intrinsic role in the development of cardiovascular disease

\section{RESULTS}

The age standardised incidence of resistant hypertension increased from 0.93 cases per 100 person years $(95 \%$ confidence interval 0.87 to 1.00 ) in 1996 to a peak level of 2.07 cases per 100 person years (2.03 to 2.12) in 2004. Incidence then decreased to 0.42 cases per 100 person years $(0.40$ to $0.44)$ in 2015 . Age standardised prevalence increased from $1.75 \%$ (95\% confidence interval $1.66 \%$ to $1.83 \%)$ in 1995 to a peak of $7.76 \%$ ( $7.70 \%$ to $7.83 \%)$ in 2007. Prevalence then plateaued and subsequently declined to $6.46 \%$ (6.38\% to $6.54 \%$ ) in 2015 .

Compared with patients aged 65-69 years, those aged 80 or more years were more likely to have prevalent resistant hypertension throughout the study period.

\section{CONCLUSIONS}

Prevalent resistant hypertension has plateaued and decreased in recent years, consistent with a decrease in incidence from 2004 onwards. Despite this, resistant hypertension is common in the UK hypertensive population. Given the importance of hypertension as a modifiable risk factor for cardiovascular disease, reducing uncontrolled hypertension should remain a population health focus.

\section{Introduction}

Uncontrolled hypertension is a leading risk factor for cardiovascular disease related morbidity and deaths. ${ }^{1}$ Hypertension is now so widely prevalent, affecting one billion people worldwide and directly responsible for more than 10 million deaths per year, that it has been declared a global public health crisis by the World Health Organization. ${ }^{12}$ Resistant hypertension is blood pressure $\geq 140 / 90 \mathrm{~mm} \mathrm{Hg}$ despite treatment with optimal doses of three different antihypertensive drugs, one of which should be a diuretic. ${ }^{3-5}$ In instances where an individual's blood pressure is at target levels but four or more antihypertensive drugs are required, resistant hypertension can also be defined. ${ }^{4}$ Those with resistant hypertension have double the risk of cardiovascular events than those without resistant hypertension, thus making them an important group to study. ${ }^{6}$

Current evidence from a systematic review and meta-analysis of 24 studies estimates the prevalence of resistant hypertension to be between $14 \%$ and $16 \%$ of all patients with hypertension, equalling 140-160 million people globally. ${ }^{7}$ These estimates may be biased upwards for two reasons. Firstly, four randomised studies were included, which likely overestimated prevalence owing to selected patients at high cardiovascular risk involved in trials. ${ }^{8}$ Secondly, of the 20 observational studies included, which should reflect the real world burden of resistant 
hypertension more so than randomised studies, few assessed adherence to antihypertensive drugs. Non-adherence has been found to be the cause of uncontrolled hypertension in as many as 50\% of patients with supposed resistant hypertension. ${ }^{9-11}$ One previous observational study, based on US claims data, estimated the incidence of resistant hypertension at $1.9 \%{ }^{6}$ However, this estimate was based on data from 2002-06 and requires updating. Additionally, assessing the burden of resistant hypertension outside settings already studied offers benefits in terms of wider generalisability. ${ }^{71-13}$

Thus an up-to-date epidemiological study on the burden of resistant hypertension, accounting for adherence to antihypertensive drug treatment is required. Accordingly, we measured the trends in incidence and prevalence of resistant hypertension among those with treated hypertension between 1995 and 2015 in the UK primary care setting.

\section{Methods}

\section{Study design and data}

We conducted a retrospective cohort study, using the Clinical Practice Research Database (CPRD-GOLD); a nationally representative repository of deidentified electronic health records from primary care in the UK. CPRD-GOLD holds data on personal information, health related behaviours, test results, diagnoses, and prescriptions for more than 11 million people in more than 670 practices across the UK since $1987 .{ }^{14}$ It is one of the largest databases of longitudinal medical records from primary care globally and has been extensively validated. ${ }^{14} 15$ Data quality are monitored by CPRD internal processes.

\section{Population}

We identified users of antihypertensive drugs between 1995 and 2015. In CPRD data, prescriptions issued by the general practitioner are automatically recorded with a product name and number. We used product numbers to identify antihypertensive drugs (see supplementary material 1) and categorised these into 14 classes: vasodilators, centrally acting agents, adrenergic blockers, $\alpha$ blockers, angiotensin converting enzyme inhibitors, angiotensin receptor blockers, renin inhibitors, thiazide diuretics, loop diuretics, potassium sparing diuretics (eg, amiloride), aldosterone antagonists (eg, spironolactone), $\beta$ blockers, calcium channel blockers, and other. Preparations of fixed dose combinations were split into their constituent active ingredients, so that patients were attributed with exposure to each different category of drug. Eligibility for the study began on the latest of: the patient's 18th birthday, the date a patient's practice was deemed to contribute "up-to-standard" data plus one year, the patient's "current registration" date plus one year, and the patients' earliest date of hypertension diagnosis. We required a diagnosis of hypertension (see Read codes in supplementary material 2) in addition to drug use because this method gives similar estimates for treated hypertension to those from the health survey for England. ${ }^{16}$ Eligibility ended on the earliest of the date on which the patient died, the date on which the patient transferred out of practice, the last data collection date from practice, and the study end date (31 December 2015).

\section{Case definition}

Clinical guidelines define resistant hypertension in two ways: uncontrolled hypertension $(\geq 140 / 90 \mathrm{~mm}$ $\mathrm{Hg}$ ) during treatment with and adherence to three concurrent antihypertensive drugs, inclusive of a diuretic, and treatment with and adherence to four antihypertensive drugs used concurrently. ${ }^{3-5}$

To identify patients who met the three drug definition we used a multistep process. Firstly, we found users of one antihypertensive drug and followed forward to assess whether they were prescribed a second drug and a third drug within different drug categories. To ensure that patients with hypertension were using three different drugs concurrently, as opposed to switching drugs, we required that patients starting a third antihypertensive drug had repeat prescriptions for all three drugs within six months of initiating the third.

Secondly, in patients who had evidence of concurrent treatment with three antihypertensive drugs, inclusive of a diuretic, we required a blood pressure reading of systolic blood pressure $\geq 140 \mathrm{~mm} \mathrm{Hg}$ or diastolic blood pressure $\geq 90 \mathrm{~mm} \mathrm{Hg}$ within 12 months of starting the third drug. ${ }^{3-5}$ Patients with blood pressure readings $<140 / 90 \mathrm{~mm} \mathrm{Hg}$ did not meet the criteria for resistant hypertension and were excluded from our case definition at this point but could be considered at a future date if new drug changes occurred. The index date-the date on which resistant hypertension could be identified-was confirmed as the later date of evidence of concurrent use of three drugs or the date of high blood pressure reading.

Thirdly, we required patients to show good adherence to their antihypertensive drug regimen. In the absence of dispensing records, which are typically used to measure adherence at the population level, we instead used prescribing records to estimate a proxy for drug adherence. Going backwards one year from the index date, we measured proxy adherence from the date of the first prescription within this one year period until the index date or until the discontinuation date of that drug, if before the index date. Discontinuation was defined as a gap of 90 or more days between the expected finishing date of a prescription and the end of the adherence observation period (ie, the index date). Using prescription dates and computed days' supply prescribed, we calculated proxy adherence as the number of days covered by the drug divided by the number of days in the observation period. We accounted for leftover days' supply from previous prescriptions by adding to the next supply. Once proxy adherence was measured for all antihypertensive drugs, we calculated an average across all drugs for each patient. ${ }^{17}$ Binary adherence was defined as an average proxy adherence of $80 \%$ or more. ${ }^{18}$ If patients 
did not meet our definition for proxy adherence, they were excluded from the case definition of resistant hypertension. We classified patients as meeting the case definition of resistant hypertension at the earliest opportunity, regardless of whether or not the patient went on to be a concurrent user of four drugs.

Patients prescribed four different antihypertensive agents, inclusive of a diuretic, were included in the case definition in a similar process. First, we required patients starting a fourth antihypertensive drug to have repeat prescriptions for all four drugs within six months of initiating the fourth drug. We did not require a blood pressure reading for those taking four drugs in concordance with accepted definitions of resistant hypertension. ${ }^{4}$ We assessed adherence in the same way as described previously. The index date was the earliest date within the six month period that there was evidence of concurrent use of four drugs.

\section{Analysis of incidence and prevalence}

We calculated annual incidence by dividing the number of new cases of resistant hypertension in each study year by the number of person years contributed by patients with treated hypertension at risk of developing resistant hypertension in that year. The denominator included patients who were excluded from the case definition on the basis of having poor adherence. We calculated annual prevalence by dividing the number of all live patients with resistant hypertension at the end of each year by the number with treated hypertension who remained eligible at the end of that year.

We calculated crude annual rates of incidence and prevalence per 100 person years or 100 people, with $95 \%$ confidence intervals. To account for a changing age structure over time, we used direct standardisation by applying age specific rates in each year to the standard population, which was the 2015 hypertensive population in CPRD. We used Poisson models with interaction terms between calendar year and sex and between calendar year and age category to assess whether the effect of sex or age on incidence and prevalence varied over time (Wald $\mathrm{P}$ values for model). Within a data driven approach, we used Joinpoint models to analyse age standardised trends over time. ${ }^{19}$ These models select an appropriate number of "turns" in trend data and give corresponding slopes to each segment in the trend. The optimal number of turns is selected by comparing permutation test results between multiple Joinpoint models.

\section{Sensitivity analyses}

We carried out three sensitivity analyses. The first was to assess the influence of defining proxy adherence at $70 \%$, and not accounting for adherence. The second assessed how the three drug definition and the four drug definition of resistant hypertension impacted on trends. Thirdly, to accommodate that different blood pressure thresholds have been used in the management of hypertension over time, and for different subgroups, we ran an analysis between 1995 and 2000 where we defined uncontrolled hypertension as blood pressure $\geq 160 / 90 \mathrm{~mm} \mathrm{Hg}^{20}$ and ran an analysis for those aged 80 or more years where we defined uncontrolled hypertension as blood pressure $\geq 150 / 90 \mathrm{~mm} \mathrm{Hg}{ }^{3}$ All analyses were carried out using Stata MP Version 14.2 and Joinpoint Regression Programme Version 4.4.0.0. ${ }^{1921}$

\section{Patient involvement}

No patients were involved in setting the research question, nor were they involved in developing plans for recruitment, design, or implementation of the study. No patients were asked to advise on interpretation or writing up of results. There are no plans to disseminate the results of the research to study participants.

\section{Results}

\section{Study population}

More than 1.3 million patients met our inclusion criteria for the study (fig 1). Of the total CPRD population, $16.46 \%$ (95\% confidence interval 15.96\% to $16.96 \%$ ) were treated for hypertension. Background hypertension closely aligned with national estimates for treated hypertension over time from health survey for England data (see supplementary material 3).

\section{Incidence}

During the study period, 90973 new cases of resistant hypertension occurred in the hypertensive population (average non-weighted incidence 1.20 per 100 person years, $95 \%$ confidence interval 0.77 to 1.79 ).

Temporal trend-incidence of resistant hypertension increased from 0.93 (0.87 to 1.00) per 100 person years in 1996 to 2.01 cases (1.96 to 2.07) in 2001 (annual percentage change 21.49\%, 95\% confidence interval $13.13 \%$ to $30.48 \%$, table 1 and supplementary material 4). Incidence peaked at 2.07 cases (95\% confidence interval 2.03 to 2.12) per 100 person years in 2004 (table 1 and supplementary material 4). Incidence then declined between 2004 and 2009 (annual percentage change $-17.61 \%$, $-21.15 \%$ to $-13.09 \%$ ) and less rapidly between 2009 and $2015(-10.19 \%,-13.62 \%$ to $-6.64 \%)$ to reach 0.42 cases ( $95 \%$ confidence interval 0.40 to 0.44 ) per 100 person years (table 1 and supplementary material 4).

\section{Age and sex}

Those aged more than 70 years were more likely than those aged 65-69 years to develop incident resistant hypertension during the study period: incidence rate ratios 1.12 (95\% confidence interval 1.09 to 1.14 ) for those aged $70-74$ years, 1.19 (1.16 to 1.23) for those aged 75-79 years, and 1.07 (1.04 to 1.10) for those aged 80 or more years (see supplementary material 5). Women were $2 \%$ less likely to develop incident resistant hypertension than men $(0.98,0.96$ to 0.99 , see supplementary material 5).

We found no evidence showing that the effect of sex or age on incidence varied over time (fig 2). 


\section{Antihypertensive drug users, 1995-2015 ( $\mathrm{n}=2237715)$}

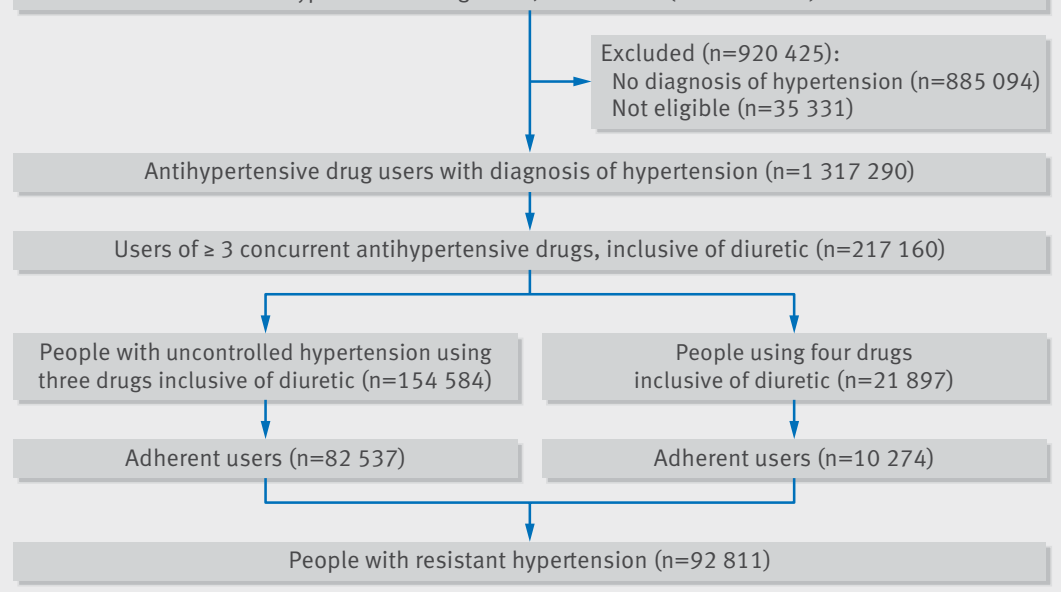

Fig 1 | Flowchart of cohort identification
Age and sex-older people were more likely to be prevalent cases. The prevalence in those aged 80 or more years was 1.43 times (95\% confidence interval 1.39 to 1.46 ) that of those aged 65-69 years (see supplementary material 5). Over time, people aged 80 or more years were more likely to have prevalent resistant hypertension with each passing year from the early 2000s onwards $(\mathrm{P}<0.001)$ (fig 3$)$. We found no evidence showing that the effect of sex on prevalence varied over time (fig 3).

\section{Sensitivity analyses}

When proxy adherence was defined at a $70 \%$ threshold, or not accounted for, trends mirrored those defined at an $80 \%$ threshold, albeit with higher peak incidences in 2004 and peak prevalences in 2007, respectively (see supplementary material 6). Trends in incidence and prevalence were driven by the three drug definition, although the prevalence of four drug use has increased over time (see supplementary material 7). When hypertension was defined using $\mathrm{a} \geq 160 / 90 \mathrm{~mm} \mathrm{Hg}$ threshold in the 1990s, we found similar trends to the main analysis but at lower absolute levels (see supplementary material 8). When hypertension was defined as $\geq 150 / 90 \mathrm{~mm}$ $\mathrm{Hg}$ for those aged 80 years or more, prevalence still increased over time, although not as dramatically as when hypertension was defined as $\geq 140 / 90 \mathrm{~mm} \mathrm{Hg}$ (see supplementary material 8).

\section{Discussion}

In this longitudinal cohort study of more than 1.3 million people treated for hypertension, the incidence of resistant hypertension increased steeply between the late 1990s and the mid-2000s, and peaked at

\begin{abstract}
change of $-3.08 \%$ (95\% confidence interval $-3.93 \%$ plateau occurred. Thereafter, prevalence decreased to 6.46 cases in 2015, with an annual percentage to $-2.22 \%, \mathrm{P}<0.001$ ) (see supplementary material 4 ).

Prevalence

prevalent cases $(95 \%$ confidence interval 1.66 to confidence interval $14.98 \%$ to $17.64 \%, \mathrm{P}<0.001$ ). This changed to $8.15 \%$ ( $4.06 \%$ to $12.40 \%, \mathrm{P}<0.001)$ between 2003 and 2006, peaking at 7.76 cases $(95 \%$ confidence interval 7.70 to 7.83 ) in 2007 before a brief
\end{abstract}

\begin{tabular}{|c|c|c|c|c|c|c|c|c|}
\hline Year & $\begin{array}{l}\text { No of incident } \\
\text { resistant } \\
\text { hypertension } \\
\text { cases }\end{array}$ & $\begin{array}{l}\text { Person } \\
\text { years }\end{array}$ & $\begin{array}{l}\text { Crude incidence } \\
\text { per } 100 \text { person } \\
\text { years }(95 \% \mathrm{Cl})\end{array}$ & $\begin{array}{l}\text { Standardised* } \\
\text { incidence per } 100 \\
\text { person years to } 2015 \\
\text { population }\end{array}$ & $\begin{array}{l}\text { No of prevalent } \\
\text { resistant } \\
\text { hypertension } \\
\text { cases }\end{array}$ & $\begin{array}{l}\text { No of prevalent } \\
\text { hypertensive } \\
\text { patients }\end{array}$ & $\begin{array}{l}\text { Crude prevalence } \\
\text { per } 100 \text { people } \\
(95 \% \mathrm{Cl})\end{array}$ & $\begin{array}{l}\text { Standardised* } \\
\text { prevalence to } 2015 \\
\text { population }\end{array}$ \\
\hline 1995 & & & & & 1657 & 90885 & 1.82 (0.95 to 2.70$)$ & 1.75 (1.66 to 1.83$)$ \\
\hline 1996 & 944 & 97842.4 & 0.96 (0.35 to 1.58$)$ & 0.93 (0.87 to 1.00$)$ & 2476 & 102534 & 2.41 (1.46 to 3.37$)$ & 2.31 (2.22 to 2.40$)$ \\
\hline 1997 & 912 & 114684.9 & $0.79(0.28$ to 1.31$)$ & 0.78 (0.72 to 0.83$)$ & 3217 & 122552 & $2.63(1.72$ to 3.53$)$ & $2.52(2.43$ to 2.60$)$ \\
\hline 1998 & 1250 & 137338.3 & 0.91 (0.41 to 1.41$)$ & 0.88 (0.83 to 0.93$)$ & 4236 & 147899 & $2.86(2.00$ to 3.73$)$ & $2.74(2.66$ to 2.82$)$ \\
\hline 1999 & 2152 & 164924.6 & $1.30(0.75$ to 1.86$)$ & $1.26(1.20$ to 1.31$)$ & 6077 & 180905 & $3.36(2.51$ to 4.20$)$ & 3.21 (3.13 to 3.29$)$ \\
\hline 2000 & 3836 & 211231.4 & 1.81 (1.24 to 2.39$)$ & $1.75(1.70$ to 1.81$)$ & 9424 & 238657 & $3.95(3.15$ to 4.75$)$ & 3.79 (3.71 to 3.86$)$ \\
\hline 2001 & 5755 & 276205.0 & 2.08 (1.54 to 2.62$)$ & 2.01 (1.96 to 2.07$)$ & 14475 & 303426 & 4.77 (3.99 to 5.55) & $4.58(4.50$ to 4.65$)$ \\
\hline 2002 & 6808 & 328707.7 & 2.07 (1.58 to 2.56$)$ & $2.00(1.96$ to 2.05$)$ & 20158 & 362534 & 5.56 (4.79 to 6.33$)$ & $5.34(5.26$ to 5.41$)$ \\
\hline 2003 & 8266 & 392679.0 & 2.10 (1.65 to 2.56$)$ & $2.04(2.00$ to 2.09$)$ & 26975 & 433536 & $6.22(5.48$ to 6.96$)$ & $5.97(5.90$ to 6.04$)$ \\
\hline 2004 & 9706 & 453736.8 & 2.14 (1.71 to 2.56$)$ & 2.07 (2.03 to 2.12$)$ & 34779 & 502592 & $6.92(6.19$ to 7.65$)$ & 6.63 (6.56 to 6.70$)$ \\
\hline 2005 & 9046 & 507018.8 & 1.78 (1.41 to 2.15$)$ & $1.73(1.70$ to 1.77$)$ & 41517 & 555801 & 7.47 (6.75 to 8.19) & 7.15 (7.08 to 7.22$)$ \\
\hline 2006 & 7684 & 532992.9 & $1.44(1.12$ to 1.76$)$ & $1.39(1.36$ to 1.43$)$ & 45993 & 579957 & $7.93(7.21$ to 8.66$)$ & $7.56(7.49$ to 7.63$)$ \\
\hline 2007 & 6638 & 548982.9 & 1.21 (0.92 to 1.50$)$ & $1.17(1.14$ to 1.20$)$ & 48720 & 594430 & $8.20(7.47$ to 8.92$)$ & $7.76(7.70$ to 7.83$)$ \\
\hline 2008 & 5424 & 562479.1 & 0.96 (0.71 to 1.22$)$ & $0.94(0.91$ to 0.96$)$ & 50074 & 613436 & $8.16(7.45$ to 8.88$)$ & $7.70(7.63$ to 7.76$)$ \\
\hline 2009 & 4630 & 572852.2 & $0.81(0.57$ to 1.04$)$ & $0.79(0.76$ to 0.81$)$ & 50093 & 618126 & $8.10(7.39$ to 8.81$)$ & 7.61 (7.54 to 7.67$)$ \\
\hline 2010 & 4253 & 572209.5 & $0.74(0.52$ to 0.96$)$ & $0.72(0.70$ to 0.74$)$ & 49080 & 612663 & $8.01(7.30$ to 8.72$)$ & 7.49 (7.42 to 7.55$)$ \\
\hline 2011 & 3656 & 562208.6 & 0.65 (0.44 to 0.86$)$ & $0.63(0.61$ to 0.65$)$ & 47624 & 601090 & $7.92(7.21$ to 8.63$)$ & 7.36 (7.29 to 7.42$)$ \\
\hline 2012 & 3245 & 557515.6 & 0.58 (0.38 to 0.78$)$ & 0.56 (0.54 to 0.58$)$ & 45680 & 589656 & $7.75(7.04$ to 8.46$)$ & 7.15 (7.08 to 7.21$)$ \\
\hline 2013 & 2752 & 525711.3 & 0.52 (0.33 to 0.72$)$ & 0.51 (0.49 to 0.53$)$ & 41409 & 543900 & $7.61(6.88$ to 8.35$)$ & $6.98(6.92$ to 7.05$)$ \\
\hline 2014 & 2243 & 476279.0 & $0.47(0.27$ to 0.66$)$ & 0.46 (0.44 to 0.48$)$ & 34678 & 477887 & 7.26 (6.49 to 8.02$)$ & $6.62(6.55$ to 6.69$)$ \\
\hline 2015 & 1773 & 406033.6 & $0.43(0.23$ to 0.63$)$ & $0.42(0.40$ to 0.44$)$ & 26877 & 379686 & $7.08(6.23$ to 7.93$)$ & $6.46(6.38$ to 6.54$)$ \\
\hline
\end{tabular}




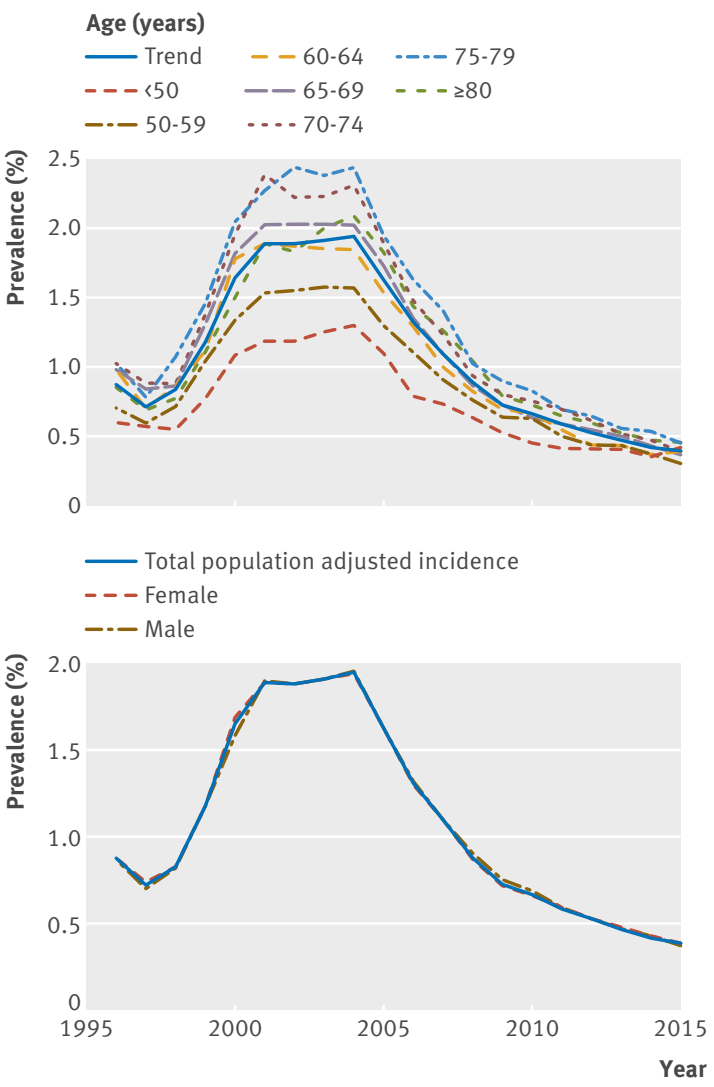

Fig 2 | Incidence stratified by age category and sex, 1996-2016

approximately two cases per 100 person years in 2004. Thereafter, the incidence decreased to 0.4 cases per 100 person years in 2015. Prevalence increased steadily from $1.8 \%$ in 1995 , peaking at $7.8 \%$ in 2007 . Thereafter, the trend declined gently, reaching $6.5 \%$ in 2015. Some evidence showed that those aged 80 or more years were more likely than those aged 65-69 years to have prevalent resistant hypertension over the study period.

\section{Strengths and weaknesses of this study}

This is the largest longitudinal study to assess the prevalence and incidence of resistant hypertension to date. We included more than 1.3 million users of antihypertensive drugs with a diagnosis of hypertension. This definition ensured the use of antihypertensive drugs in those with hypertension, although not all blood pressure lowering drugs may have been intended for the treatment of hypertension. None the less, the prevalence of treated hypertension in our study closely matched national prevalence estimates for treated hypertension in the health survey for England (see supplementary material 3). In the absence of dispensing data, we used prescribing data within CPRD to estimate a proxy measure of adherence. Compared with dispensing data, prescribing data can overestimate adherence to cardiovascular drugs, from $5 \%{ }^{22}$ to $20 \% .^{23}$ This may have impacted our results by overestimating
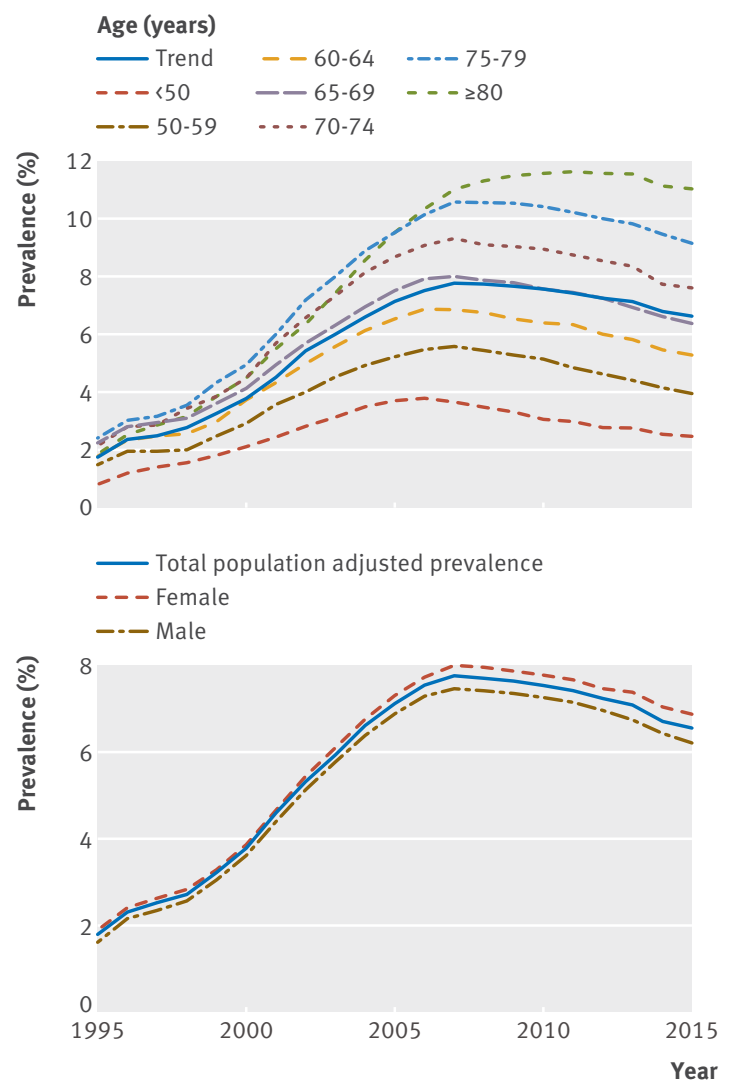

Fig 3 | Prevalence stratified by age category and sex, 1995-2016

the number of patients who were adherent, thus inflating prevalence and incidence. Assuming a worst case scenario whereby our figures represent an overestimation of $20 \%$, peak prevalence would then be approximately $6 \%$ and peak incidence would be approximately 1.7 cases per 100 person years. Thus we acknowledge the limitations of our method but believe our estimates are strengthened by using what was the only available approach for assessing adherence in our population level data source. Furthermore, our various sensitivity analyses on adherence give a range of estimates to inform interpretation. We used clinic blood pressure measurements in this study, which are susceptible to white coat hypertension. However, we believe the trends we have reported are reflective of real world patterns, based on treatment decisions and management pathways as they occur in reality in patients with established hypertension. We used one blood pressure measurement to ascertain whether patients had controlled or uncontrolled hypertension. Previous research found that blood pressure measurements in electronic health records are representative of adjacent measurements ${ }^{24}$ and that using two measurements confers no advantage over one measurement in identifying patients with resistant hypertension. ${ }^{25}$ Another source of pseudoresistant hypertension is secondary causes; we found these to be negligibly distributed among those with resistant hypertension (see supplementary material 9). 
We used a general threshold of blood pressure $\geq 140 / 90 \mathrm{~mm} \mathrm{Hg}$ to define hypertension at the population level, which is the accepted threshold in national and international guidelines. ${ }^{3} 526$ This approach was pragmatic given changing threshold values for the general population and for clinical subgroups during the study period. ${ }^{3} 52026-28$ We tested the appropriateness of this approach with two sensitivity analyses, using a $\geq 160 / 90 \mathrm{~mm} \mathrm{Hg}$ threshold for defining hypertension between 1995 and 2000 and $\mathrm{a} \geq 150 / 90 \mathrm{~mm} \mathrm{Hg}$ threshold for defining hypertension for those aged 80 or more years, both of which indicated broadly similar trends to the main analysis. We did not require "optimal" doses of each antihypertensive drug because this feature of prescribing is highly individualised, especially in patients who use polypharmacy. Rather, we focused on establishing population trends using population level data.

\section{Relation to other studies and key differences}

One previous study, using three cross sections of the American National Health and Nutritional Examination Survey examined prevalence of resistant hypertension over time. ${ }^{29}$ This study reported a linear increase in the prevalence of resistant hypertension among all patients with hypertension (treated and untreated), ranging from $5.5 \%$ in $1988-94$ to $8.5 \%$ in $1999-2004$ to $11.8 \%$ in $2005-08$. This steady incline in prevalence mirrors the incline we noted during similar timeframes, although we observed a peak prevalence of almost 8\% in 2007 and a plateau and decline thereafter. Our estimates are likely lower because we accounted for adherence in our case definition, whereas this was not possible in the American study. The same reason is plausibly responsible for the difference between a summary prevalence estimate of $14 \%$ based on 20 observational studies in a recent systematic review and our peak prevalence estimate of $8 \% .^{7}$ Many of the studies included in the review did not account for adherence. In our sensitivity analysis, which did not account for adherence, we found a prevalence of approximately $14 \%$ in the past 10 years (see supplementary material 6 ), thus agreeing with the results reported in the systematic review and recent similar additions to the literature from Africa ${ }^{11}$ and Germany. ${ }^{12}$ By contrast, our results for prevalent resistant hypertension (accommodating for adherence) agree well with prevalence estimates from the 2011 health survey for England. ${ }^{30}$

We found some evidence showing that those aged 80 or more years were more likely to have prevalent resistant hypertension over the study period compared with those aged 65-69 years, a finding that was robust to sensitivity analyses. This adds to evidence from Sweden in which older patients had a higher prevalence of resistant hypertension than their younger counterparts according to various definitions of resistant hypertension. ${ }^{13}$

Data from the Anglo Scandinavian Cardiac Outcomes Trial (ASCOT) estimated incidence of resistant hypertension at approximately 34\%, which is a probable overestimate owing to nonrepresentativeness of the clinical trial population. ${ }^{31}$ In contrast with ASCOT data, an observational study found an incidence of approximately $2 \%$ based on American administrative claims data between 2002 and 2006, which is more similar to the peak incidence of just over two cases per 100 person years we found in $2004 .^{6}$

\section{Meaning of findings and possible mechanisms}

The explanation for the trends we found is likely multifactorial, relating to detection, treatment, and patient awareness of hypertension as opposed to changing pathology. The steep increase in incidence between 1996 and 2004 might be explained by increased detection and treatment of hypertension during this period, resulting from a burgeoning interest in this clinical area and the publication of several seminal clinical trials. ${ }^{32-36}$ Increasingly aggressive treatment was supported by evidence from the Antihypertensive and Lipid-Lowering Treatment to Prevent Heart Attack Trial (ALLHAT) that most patients needed at least two antihypertensive drugs to achieve target blood pressure levels. ${ }^{37}$ The initiation of the Quality and Outcomes Framework in the UK may partially explain the fall-off in incidence after 2004. This pay for performance scheme incentivised general practitioners to more closely monitor blood pressure levels and to increase the percentage of patients with controlled ( $<150 / 90 \mathrm{~mm} \mathrm{Hg}$ ) hypertension. A report on the impact of the framework using QRESEARCH data from primary care found that rates of blood pressure control ( $<150 / 90 \mathrm{~mm} \mathrm{Hg}$ ) increased by $65 \%$ between 2001 and 2006. ${ }^{38}$ Control rates defined as blood pressure $<140 / 90 \mathrm{~mm} \mathrm{Hg}$ increased from 5.7\% in 2003 to $9.9 \%$ in $2014 .{ }^{39}$ Throughout the study period, the proportion of patients aware of their hypertension, and possibly the importance of its treatment, also increased, ${ }^{30}$ perhaps also playing a contributory role in improved control rates.

\section{Conclusions and implications for clinicians and policy makers}

Resistant hypertension is common in the UK hypertensive population. Given the importance of hypertension as a modifiable risk factor for cardiovascular disease, ${ }^{40}$ continued efforts are warranted to reduce the proportion of the population with uncontrolled hypertension. Points for intervention include frequent checks on drug adherence, considering its role in causation of resistant hypertension, and review of drug regimens. ${ }^{3}$ Where possible, once daily dosing should be used, along with fixed dose combination preparations to help improve adherence. ${ }^{4142}$ Self management and shared management between general practitioners and other health professionals such as nurses and pharmacists can also help improve blood pressure control. ${ }^{43} 44$ Educational interventions targeted at patients may help to improve awareness and encourage adherence. ${ }^{45}$ Our 
results are generalisable within the UK and also bear similarities to estimates from various other countries. However, differences in the organisation of care and the implementation of policy initiatives likely lead to a unique pattern of trends in the UK.

Contributors: SJS, LS, EW, and IJD were the sole contributors and authors of this study. All authors played a substantial role in developing the research question and writing the protocol, obtaining the data, interpreting the results, and preparing the manuscript. SJS carried out the analysis. SJS is guarantor.

Funding: SJS is supported by a Wellcome Trust Sir Henry Wellcome fellowship (107340/Z/15/Z). LS is supported by a Wellcome Trust senior research fellowship in clinical science (098504/Z/12/Z). IJD is supported by an unrestricted grant from GlaxoSmithKline. The funders had no role in the design or conduct of this work.

Competing interests: All authors have completed the ICMJE uniform disclosure form at www.icmje.org/coi_disclosure.pdf and declare: LS reports grants from the Wellcome Trust and British Heart Foundation during the conduct of the study; grants from the Wellcome Trust, Medical Research Council, National Institute for Health Research, and the European Union outside the submitted work personal fees from GlaxoSmithKline for advisory work unrelated to the submitted work; grant funding from GlaxoSmithKline for academic research unrelated to the submitted work; acts as an unpaid steering committee chair for AstraZeneca for a randomised trial unrelated to the submitted work; and is a trustee of the British Heart Foundation; IJD is funded by an unrestricted grant from GlaxoSmithKline, has consulted for GlaxoSmithKline and Gilead, and holds stock in GlaxoSmithKline.

Ethical approval: The study protocol was approved by the London School of Hygiene and Tropical Medicine ethics committee (No 13988) and the independent scientific advisory committee for Medicines and Healthcare products Regulatory Agency (No 16_205). The protocol was made available to reviewers as supplementary material.

Data sharing: No additional data available.

Transparency: The manuscript's guarantor (SJS) affirms that the manuscript is an honest, accurate, and transparent account of the study being reported; that no important aspects of the study have been omitted; and that any discrepancies from the study as planned have been explained.

This is an Open Access article distributed in accordance with the terms of the Creative Commons Attribution (CC BY 4.0) license, which permits others to distribute, remix, adapt and build upon this work, for commercial use, provided the original work is properly cited. See: http://creativecommons.org/licenses/by/4.0/.

1 Forouzanfar MH, Afshin A, Alexander LT, et al. GBD 2015 Risk Factors Collaborators. Global, regional, and national comparative risk assessment of 79 behavioural, environmental and occupational, and metabolic risks or clusters of risks, 1990 2015: a systematic analysis for the Global Burden of Disease Study 2015. Lancet 2016;388:1659-724. doi:10.1016/S01406736(16)31679-8

2 World Health Organisation (WHO). A Global Brief on Hypertension: Silent Killer, Global Public Health Crisis [Online]. Available from http:// www.who.int/cardiovascular_diseases/publications/global_brief_ hypertension/en/. 2013

3 National Institute for Health and Care Excellence. Hypertension in adults: diagnosis and management. NICE guidelines [CG127], 2011.

4 Calhoun DA, Jones D, Textor S, et al. American Heart Association Professional Education Committee. Resistant hypertension: diagnosis, evaluation, and treatment. Circulation 2008;117: e510-26. doi:10.1161/CIRCULATIONAHA.108.189141

5 Mancia G, Fagard R, Narkiewicz K, et al. Task Force for the management of arterial hypertension of the European Society of Hypertension. Task Force for the management of arterial hypertension of the European Society of Cardiology. 2013 ESH/ ESC Guidelines for the Management of Arterial Hypertension. Blood Press 2013;22:193-278. doi:10.3109/08037051.2013.812549

6 Daugherty SL, Powers JD, Magid DJ, et al. Incidence and prognosis of resistant hypertension in hypertensive patients. Circulation 2012;125:1635-42. doi:10.1161/ CIRCULATIONAHA.111.068064

7 Achelrod D, Wenzel U, Frey S. Systematic review and metaanalysis of the prevalence of resistant hypertension in treated hypertensive populations[published Online First: 2014/08/27]. Am Hypertens 2015;28:355-61. doi:10.1093/ajh/hpu151.
8 Pimenta E, Calhoun DA. Resistant hypertension: incidence, prevalence, and prognosis. Circulation 2012;125:1594-6. doi:10.1161/CIRCULATIONAHA.112.097345.

9 Jung O, Gechter JL, Wunder C, et al. Resistant hypertension? Assessment of adherence by toxicological urine analysis. / Hypertens 2013;31: 766-74. doi:10.1097/ HJH.Ob013e32835e2286

10 Tomaszewski M, White C, Patel P, et al. High rates of non-adherence to antihypertensive treatment revealed by high-performance liquid chromatography-tandem mass spectrometry (HP LC-MS/MS) urine analysis. Heart 2014;100:855-61.

11 Nansseu JR, Noubiap JJ, Mengnjo MK, et al. The highly neglected burden of resistant hypertension in Africa: a systematic review and meta-analysis[published Online First: 2016/09/22] BMJ Open 2016;6:e011452. doi:10.1136/bmjopen2016-011452.

12 Sarganas G, Neuhauser HK. Untreated, uncontrolled, and apparent resistant hypertension: results of the German Health Examination Survey 2008-2011. J Clin Hypertens (Greenwich) 2016;18:1146 54. doi:10.1111/jch.12886

13 Holmqvist L, Boström KB, Kahan T, et al. Prevalence of treatmentresistant hypertension and important associated factors-results from the Swedish Primary Care Cardiovascular Database. J Am Soc Hypertens 2016;10:838-46. doi:10.1016/j.jash.2016.08.008

14 Herrett E, Gallagher AM, Bhaskaran K, et al. Data resource profile: clinical practice research datalink (CPRD). Int I Epidemiol 2015;44:827-36. doi:10.1093/ije/dyv098

15 Herrett E, Shah AD, Boggon R, et al. Completeness and diagnostic validity of recording acute myocardial infarction events in primary care, hospital care, disease registry, and national mortality records: cohort study. BMJ 2013;346:f2350. doi:10.1136/bmj.f2350

16 Ryan R, Majeed A. Prevalence of treated hypertension in general practice in England and Wales, 1994 to 1998. Health Stat Q 2002;(16):14-8

17 Choudhry NK, Shrank WH, Levin RL, et al. Measuring concurrent adherence to multiple related medications. Am J Manag Care 2009; 15:457-64

18 Karve S, Cleves MA, Helm M, Hudson TJ, West DS, Martin BC. Good and poor adherence: optimal cut-point for adherence measures using administrative claims data. Curr Med Res Opin 2009;25:2303-10. doi:10.1185/03007990903126833

19 National Cancer Institute. Joinpoint Regression Program, Version 4.4.0.0 Statistical Methodology and Applications Branch, Surveillance Research Program. National Cancer Institute, 2017

20 Sever P, Beevers G, Bulpitt C, et al. Management guidelines in essential hypertension: report of the second working party of the British Hypertension Society. BMJ 1993;306:983-7. doi:10.1136/ bmj.306.6883.983

21 Stata Statistical Software. Release 14. [program] StataCorp LP, 2015.

22 Pottegård A, Christensen Rd, Houji A, et al. Primary non-adherence in general practice: a Danish register study[published Online First: 2014/04/24]. Eur / Clin Pharmacol 2014;70:757-63. doi:10.1007/ s00228-014-1677-y.

23 Fischer MA, Stedman MR, Lii J, et al. Primary medication nonadherence: analysis of 195,930 electronic prescriptions. I Gen Intern Med 2010;25:284-90. doi:10.1007/s11606-010-1253-9.

24 Hasselström J, Zarrinkoub R, Holmquist C, et al. The Swedish Primary Care Cardiovascular Database (SPCCD): 74751 hypertensive primary care patients. Blood Press 2014:23:116-25 doi:10.3109/0803705 1.2013.814829

25 McAdam-Marx C, Ye X, Sung JC, Brixner DI, Kahler KH. Results of a retrospective, observational pilot study using electronic medical records to assess the prevalence and characteristics of patients with resistant hypertension in an ambulatory care setting. Clin Ther 2009;31:1116-23. doi:10.1016/ j.clinthera.2009.05.007

26 James PA, Oparil S, Carter BL, et al. 2014 evidence-based guideline for the management of high blood pressure in adults: report from the panel members appointed to the Eighth Joint National Committee (JNC 8). JAMA 2014;311:507-20. doi:10.1001/jama.2013.284427

27 Ramsay LE, Williams B, Johnston GD, et al. British Hypertension Society guidelines for hypertension management 1999: summary. BMJ 1999;319:630-5. doi:10.1136/bmj.319.7210.630

28 Chobanian AV, Bakris GL, Black HR, et al. National Heart, Lung, and Blood Institute Joint National Committee on Prevention, Detection, Evaluation, and Treatment of High Blood Pressure, National High Blood Pressure Education Program Coordinating Committee. The seventh report of the joint national committee on prevention, detection, evaluation, and treatment of high blood pressure: the JNC 7 report. JAMA 2003;289:2560-72. doi:10.1001/ jama.289.19.2560

29 Egan BM, Zhao Y, Axon RN, Brzezinski WA, Ferdinand KC. Uncontrolled and apparent treatment resistant hypertension in the United States, 1988 to 2008. Circulation 2011;124:1046-58. 
30 Falaschetti E, Mindell J, Knott C, Poulter N. Hypertension management in England: a serial cross-sectional study from 1994 to 2011 Lancet 2014;383:1912-9. doi:10.1016/S0140-6736(14)60688-7

31 Gupta AK, Nasothimiou EG, Chang CL, Sever PS, Dahlöf B, Poulter NRASCOT investigators. Baseline predictors of resistant hypertension in the Anglo-Scandinavian Cardiac Outcome Trial (ASCOT): a risk score to identify those at high-risk. J Hypertens 2011;29:2004-13. doi:10.1097/ HIH.Ob013e32834a8a42

32 UK Prospective Diabetes Study Group. Tight blood pressure contro and risk of macrovascular and microvascular complications in type 2 diabetes: UKPDS 38. BMJ 1998;317:703-13.

33 Staessen JA, Fagard R, Thijs L, et al. The Systolic Hypertension in Europe (Syst-Eur) Trial Investigators. Randomised double-blind comparison of placebo and active treatment for older patients with isolated systolic hypertension[published Online First: 1997/09/23]. Lancet 1997;350:757-64. doi:10.1016/S01406736(97)05381-6

34 Hansson L, Zanchetti A, Carruthers SG, et al. HOT Study Group. Effects of intensive blood-pressure lowering and low-dose aspirin in patients with hypertension: principal results of the Hypertension Optimal Treatment (HOT) randomised trial. Lancet 1998;351:175562. doi:10.1016/S0140-6736(98)04311-6

35 Perry HMJr, Davis BR, Price TR, et al. Effect of treating isolated systolic hypertension on the risk of developing various types and subtypes of stroke: the Systolic Hypertension in the Elderly Program (SHEP). JAMA 2000;284:465-71. doi:10.1001/jama.284.4.465

36 ALLHAT Officers and Coordinators for the ALLHAT Collaborative Research Group. The Antihypertensive and Lipid-Lowering Treatment to Prevent Heart Attack Trial. Major outcomes in moderately hypercholesterolemic, hypertensive patients randomized to pravastatin vs usual care: The Antihypertensive and Lipid-Lowering Treatment to Prevent Heart Attack Trial (ALLHAT-LLT). JAMA 2002;288:2998-3007. doi:10.1001/ jama.288.23.2998

37 Cushman WC, Ford CE, Cutler JA, et al. ALLHAT Collaborative Research Group. Success and predictors of blood pressure control in diverse North American settings: the antihypertensive and lipid-lowering treatment to prevent heart attack trial (ALLHAT)[published Online First: 2002/12/04]. J Clin Hypertens (Greenwich) 2002;4:393-404. doi:10.1111/j.1524-6175.2002.02045x

38 Hippisley-Cox J, Vinogradova Y, Coupland C. Time Series Analysis for selected clinical indicators from the Quality and Outcomes Framework 2001-2006. The Information Centre for Health and Social Care, 2007.

39 Health Survey for England. Trend Tables: Table 1 Blood Pressure leve using Omron values and 2003 definition by survey year, age and sex. Available at http://content.digital.nhs.uk/catalogue/PUB19297, 2014.

40 Ettehad D, Emdin CA, Kiran A,et al. Blood pressure lowering for prevention of cardiovascular disease and death: a systematic review and meta-analysis. Lancet 2016;387:957-67. doi:10.1016/S0140 6736(15)01225-8

41 Schroeder K, Fahey T, Ebrahim S. How can we improve adherence to blood pressure-lowering medication in ambulatory care? Systematic review of randomized controlled trials. Arch Intern Med 2004:164:722-32. doi:10.1001/archinte.164.7.722

42 Gupta AK, Arshad S, Poulter NR. Compliance, safety, and effectiveness of fixed-dose combinations of antihypertensive agents: a meta-analysis. Hypertension 2010;55:399-407. doi:10.1161/ HYPERTENSIONAHA.109.139816

43 Walsh JM, McDonald KM, Shojania KG, et al. Quality improvement strategies for hypertension management: a systematic review. Med Care 2006:44:646-57 doi:10.1097/01. mlr.0000220260.30768.32

44 Glynn LG, Murphy AW, Smith SM, Schroeder K, Fahey T. Interventions used to improve control of blood pressure in patients with hypertension. Cochrane Database Syst Rev 2010;(3):CD005182.

45 Ribeiro CD, Resqueti VR, Lima Í, et al. Educational interventions for improving control of blood pressure in patients with hypertension: a systematic review protocol. BMJ Open 2015;5(3):e006583.

Supplementary material: supplementary material 1 and 2

Supplementary material: supplementary material 3-9 